\title{
Discours
}

Revue de linguistique, psycholinguistique et

informatique. A journal of linguistics, psycholinguistics and computational linguistics

$25 \mid 2019$

Varia

Chaînes de référence et structure textuelle dans les Essais sur la peinture de Diderot

Céline Guillot-Barbance et Matthieu Quignard

URL : http://journals.openedition.org/discours/10421

DOI : $10.4000 /$ discours. 10421

ISSN : 1963-1723

Éditeur :

Laboratoire LATTICE, Presses universitaires de Caen

Référence électronique

Céline Guillot-Barbance et Matthieu Quignard, « Chaînes de référence et structure textuelle dans les Essais sur la peinture de Diderot », Discours [En ligne], 25 | 2019, mis en ligne le 30 décembre 2019, consulté le 04 avril 2020. URL : http://journals.openedition.org/discours/10421 ; DOI : https://doi.org/ 10.4000/discours. 10421 

Revue de linguistique, psycholinguistique et informatique

\section{Chaînes de référence et structure textuelle dans les Essais sur la peinture de Diderot}

Céline Guillot-Barbance

UMR 5371 IRHIM (Institut d'histoire des représentations et des idées dans les modernités), ENS de Lyon

Matthieu Quignard

UMR 5191 ICAR (Interactions, corpus, apprentissages, représentations), CNRS

Céline Guillot-Barbance, Matthieu Quignard, «Chaînes de référence et structure textuelle dans les Essais sur la peinture de Diderot», Discours [En ligne], 25 | 2019, mis en ligne le 30 décembre 2019.

URL: http://journals.openedition.org/discours/10421

Titre du numéro: Varia

Coordination: Laure Sarda \& Denis Vigier

Date de réception de l'article: 27/07/2019

Date d'acceptation de l'article: 15/12/2019 



\title{
Chaînes de référence et structure textuelle dans les Essais sur la peinture de Diderot
}

\author{
Céline Guillot-Barbance \\ UMR 5371 IRHIM (Institut d'histoire des représentations et des idées dans les modernités), ENS de Lyon \\ Matthieu Quignard \\ UMR 5191 ICAR (Interactions, corpus, apprentissages, représentations), CNRS
}

\begin{abstract}
Nous étudions le rapport qu'entretiennent les chaînes de référence avec la structure textuelle des trois premiers chapitres des Essais sur la peinture de Diderot. Le corpus est issu du projet ANR DEMOCRAT au sein duquel il a été préparé et annoté. Dans une première partie, nous analysons la distribution des chaînes au sein des unités structurelles (chapitres et paragraphes), afin d'observer leur variabilité en termes de longueur et de couverture (densité référentielle et empan). Dans une seconde partie, nous nous intéressons plus précisément au rapport qu'on peut établir entre les chaînes et le thème développé dans les unités structurelles. Les analyses quantitatives multi-niveaux menées à l'aide de l'extension «Annotation URS» du logiciel TXM indiquent que les chaînes de référence jouent un rôle dans le marquage de la continuité et de la discontinuité textuelle. Le thème des chapitres est plutôt associé aux chaînes longues, tandis que le thème des paragraphes s'exprime par des chaînes courtes et souvent discontinues par rapport à celles des autres paragraphes (nouvelles et spécifiques au paragraphe d'occurrence).
\end{abstract}

Mots clés: chaînes de référence, structures textuelles, thème de discours, corpus annoté, textométrie

This paper addresses the relation between reference chains and structural units, in the three first chapters of the Essais sur la peinture by Diderot. This corpus is taken from the DEMOCRAT project, during which it has been prepared and annotated. In a first part, a close analysis addresses the distribution of referents and chains across the three chapters and five comparable paragraphs, in order to observe the variability in lengths, coverage and density. A second part focuses on reference chains with respect to units theme (chapter titles, paragraph themes). Multilevel quantitative analyses have been operated with help of the URS extension of TXM. It appears that reference chains do affect textual continuity and discontinuity. More precisely, chapter themes are rather marked by long and continuous chains whereas paragraph ones are rather marked by short and discontinuous chains (new, local and specific to that unit).

Keywords: reference chains, textual structures, discourse theme, annotated corpus, textometry

Nous tenons en tout premier lieu à remercier les relecteurs de la revue Discours pour la qualité et la précision de leurs remarques qui nous ont permis d'améliorer le présent article. Notre gratitude va également à nos collègues du projet DEMOCRAT («Description et modélisation des chaînes de référence : outils pour l'annotation de corpus et le traitement automatique »), notamment Matthieu Decorde, Serge Heiden et Bénédicte Pincemin pour toute l'aide apportée à notre recherche et aux annotatrices Zeina Tmart et Audrey Arpin-Pont pour leur travail si précieux. 


\section{Introduction}

L'analyse des chaînes de référence a connu ces dernières années un essor important en France grâce à la constitution et à la diffusion de ressources annotées, notamment le corpus ANCOR («Anaphore et coréférence dans les corpus oraux») pour le français parlé et le corpus DEMOCRAT pour le français écrit ${ }^{1}$, ainsi qu'au développement d'outils adaptés à leur exploitation ${ }^{2}$. Si l'exploitation de vastes corpus numériques semble particulièrement appropriée pour l'étude de phénomènes textuels de ce type, elle pose cependant des défis qui sont difficiles à relever sur les plans technique, méthodologique et scientifique.

Les recherches menées sur les corpus écrits ont déjà permis de dégager de grandes tendances. La plupart des travaux ont porté sur des textes entiers, souvent courts, ou sur des extraits de taille assez importante pour rendre possible l'analyse des chaînes (désormais CR) en contexte. Ces études ont mis en évidence les spécificités de certains textes ou de certains types de textes relativement à d'autres, en prenant le texte ou l'extrait comme une seule unité. Ce sont les variations diachroniques, interlinguistiques et génériques qui ont été principalement analysées (Landragin et Schnedecker, 2014; Schnedecker, 2014; Obry et al., 2017).

D’autres études, parfois plus anciennes et portant plus généralement sur la référence et l'anaphore, ont relevé l'importance des frontières textuelles dans le choix des expressions référentielles (notamment Ariel, 1990). On s'intéresse en général à ce qui se passe au début ou à la fin des unités, en faisant l'hypothèse que le passage d'une unité à l'autre a une influence sur les $\mathrm{CR}$ ou que les $\mathrm{CR}$ marquent, ou concourent à marquer, ce passage. Ce sont les catégories grammaticales des expressions référentielles qui sont alors examinées, certaines expressions marquant la continuité référentielle et textuelle, tandis que d'autres indiquent au contraire une forme de discontinuité.

On a donc pris jusqu'ici le texte comme une unité relativement homogène dans les types de CR qu'il instancie, même si l'on admet que certains lieux comme les frontières d'unités structurelles ont un impact, qu'on suppose récurrent à l'échelle de l'unité-texte, sur ces chaînes. Or, même si l'on s'en tient à des caractéristiques assez générales comme le nombre, la longueur ou la composition des $\mathrm{CR}$, rien ne dit qu'il ne puisse y avoir des variations assez importantes selon les parties internes d'un texte. Ce sera notre premier angle d'approche des interférences entre les chaînes et la structure textuelle.

On s'intéressera ensuite aux rapports complexes qu'il est possible d'établir entre thèmes de discours, $\mathrm{CR}$ et structure du texte. L'étude d'un texte organisé en deux niveaux hiérarchiques d'unités structurelles (chapitres et paragraphes), nous permettra d'étudier les chaînes dans leur relation avec l'identification du thème et avec la diversité thématique des niveaux textuels où ces chaînes se trouvent.

1. Voir: https://www.ortolang.fr/market/corpora/ortolang-000903 et http://www.lattice.cnrs.fr/democrat.

2. TXM: http://textometrie.ens-lyon.fr. 


\section{Marques configurationnelles, chaînes de référence et thèmes de discours}

Dans ses travaux fondateurs sur les plans d'organisation du texte, Charolles (1988 et 1995) définit un certain nombre d'outils relationnels de nature sémantico-pragmatique qui créent des connexions et participent à la construction de la cohérence textuelle. Ces éléments sont de différents types et agissent à différents niveaux du texte. On distingue ainsi les connecteurs, les anaphores et $\mathrm{CR}$, les expressions introductrices de cadres de discours et les marques configurationnelles, comme le paragraphe, «qui délimitent au sein du continuum textuel des ensembles présentés par le locuteur comme constituant une ou plusieurs unités en regard d'un certain critère dispositionnel» (Charolles, 1995: 128). Parmi tous ces éléments, les CR semblent jouer un rôle de premier plan dans la mesure où elles sont intimement liées au thème (ou topique) de discours.

Bien qu'elles paraissent de prime abord jouer au niveau (typo)graphique, les marques configurationnelles sont en réalité elles aussi en relation étroite avec le thème et la progression thématique. Les nombreuses études portant sur le paragraphe (notamment Longacre, 1979; Mitterand, 1985; Bessonnat, 1988; Adam, 2018), qu'elles reposent sur une approche strictement textuelle ou plus cognitive, insistent en général sur l'adéquation entre unité paragraphique et unité thématique.

Il semble donc pertinent de s'interroger sur la façon dont les CR et paragraphes interagissent pour déterminer l'organisation thématique du texte. L'un des intérêts de cette recherche est de mettre en regard des marques opérant sur un plan microtextuel, les CR, avec d'autres marques caractéristiques du niveau méso-textuel, les paragraphes (Adam, 2018). On complétera l'analyse en prenant en compte un troisième organisateur textuel, la division en chapitres, qui fait également partie des marques configurationnelles mais se situe au niveau de la macro-structure. L'autre apport des chapitres pour notre étude sera qu'ils sont introduits par des titres, dont la fonction est également thématique.

L'étude des CR dans chaque palier ou unité structurelle des Essais sur la peinture de Diderot (texte au niveau macro > chapitre au niveau macro > paragraphe au niveau méso) permettra de répondre à deux objectifs principaux: (i) mesurer le degré d'homogénéité du texte à travers l'homogénéité des CR de ses unités structurelles (section 4), (ii) vérifier dans quelle mesure les $\mathrm{CR}$ et leurs propriétés permettent d'identifier les thèmes de ces unités (section 5). On s'appuiera pour cela sur l'annotation des CR d'une partie des Essais (environ 9000 mots). L'annotation et les outils d'analyse mis en œuvre pour cette recherche ont été produits par le projet ANR DEMOCRAT ${ }^{3}$ et sont librement accessibles ${ }^{4}$.

3. DEMOCRAT - ANR-15-CE38-0008.

4. Le corpus et les outils DEMOCRAT sont entreposés sur la plateforme Ortolang: https://hdl.handle. net/11403/democrat. L'outil d'annotation et d'exploitation est accessible sous la forme d'un module d'extension appelé «Annotation URS (Unité-Relation-Schéma)» (Heiden, 2019) du logiciel TXM (Heiden et al., 2010). 


\section{Méthodologie de linguistique de corpus}

\subsection{Choix du corpus}

Le texte choisi pour cette étude, les Essais sur la peinture de Diderot, a été composé pour l'essentiel en 1766. Il est adressé à Melchior Grimm, qui le publie dans la Correspondance littéraire (revue manuscrite à diffusion très restreinte) en août, novembre et décembre 1766. C'est au même destinataire que sont adressés les Salons de 1759, 1761, 1763, 1765 et 1766 et tous ces textes paraissent dans la même revue.

Les Essais se présentent comme une sorte de complément au Salon de 1765. Diderot annonce à la fin du texte «un petit traité de peinture» qui doit «exposer franchement les motifs de confiance qu'on peut avoir dans nos jugements». Ce ne sera finalement pas un traité mais des essais qui paraitront peu après. On verra que le titre et le genre de l'œuvre peuvent faire débat mais le lien avec les Salons, sur le fond comme sur la forme, est évident.

Le texte des Essais comporte au départ cinq chapitres, qui ne sont pas numérotés mais qui sont précédés d'un titre. S'y ajoutent rapidement deux chapitres portant sur l'architecture puis un supplément au troisième chapitre, intitulé «Examen du clair-obscur». Ce supplément a sans doute été rédigé par Diderot entre 1766 et 1773. Il est absent de la première édition, qui paraitt en 1795, mais inclus dans la seconde, publiée en 1798. Les quatre chapitres annotés dans le cadre du projet DEMOCRAT sont les trois premiers chapitres initiaux et cet ajout sur le clair-obscur.

La version du texte exploitée dans cette étude a été éditée par Gita May. Elle est tirée de l'édition de référence des œuvres complètes de Diderot, dite «édition Dieckmann-Varloot», et se fonde sur les meilleures copies des Essais, principalement les copies de Stockholm. L'éditrice indique que les graphies des noms propres et la ponctuation du texte original ont été respectées tandis que l'orthographe a été modernisée. Les limites des structures textuelles (chapitres et paragraphes) varient très peu entre cette version et la première édition des Essais. Seul le paragraphe 14 de l'édition moderne regroupe deux paragraphes du texte imprimé en 1795. Puisque les structures textuelles qui nous intéressent sont très stables entre ces deux éditions, nous pouvons nous appuyer sur l'édition May pour notre étude. Nous écartons cependant le supplément sur le clair-obscur, parce que c'est un ajout postérieur et parce que ce passage n'a pas pu être comparé avec l'édition initiale de 1795.

Du point de vue générique, le texte des Essais occupe une place très singulière. On sait que Diderot renouvelle le genre naissant de la critique d'art grâce à la «polygénéricité» de ses textes et à ce que l'on a parfois appelé «l'absence d'œuvre» (Benrekassa, 1992). Textes de commande de son ami Melchior Grimm, les Salons prennent dès le départ la forme de lettres adressées à cet ami. C'est donc sur le mode de la conversation épistolaire que sont construits à la fois les Salons et les Essais. Et la revue qui les accueille, la Correspondance littéraire, a pour vocation de rendre compte de l'actualité intellectuelle et artistique parisienne à un public très ciblé de princes européens (Fernandes, 2014). 
L'influence du genre épistolaire explique quelques-uns des traits saillants des Essais: fréquence des adresses au destinataire ${ }^{5}$, des interrogations, des verbes à l'impératif et au présent de l'indicatif, et plus généralement de tout ce qui donne l'illusion de la communication directe et de l'échange privé. Le registre familier, le ton très libre et parfois décousu qui caractérisent le texte vont dans le même sens.

L'autre trait distinctif des Essais est qu'ils mettent en scène une forme d'échange entre l'auteur et le ou les destinataire(s). De même qu'il lui arrive parfois de faire parler les tableaux dans les Salons, Diderot tend à mettre en scène une situation de débat où des avis contradictoires s'opposent et se répondent les uns aux autres. C'est bien entendu son avis et son sentiment personnel qui priment et la première personne est omniprésente dans tout le texte. La visée argumentative de l'œuvre, qui doit convaincre le lecteur du bien-fondé de ses jugements sur la peinture, est supportée par cette représentation de la parole de l'autre. Le texte prolonge ainsi à sa façon la longue tradition du dialogue didactique.

Enfin, sur le fond comme sur la forme, le but affiché de Diderot semble être de déconstruire les canons littéraires et l'académisme sclérosé. Cette déconstruction passe par exemple par la forme des titres de ses chapitres, qui cadre mal avec l'image habituelle du traité ou de l'essai : «Mes pensées bizarres sur le dessin», «Mes petites idées sur la couleur», «Tout ce que j'ai compris de ma vie du clair-obscur», etc. De même qu'il valorise la «peinture de genre», qui délaisse les grandes figures antiques et historiques au profit des thèmes de la vie quotidienne, Diderot s'écarte du grand style. En multipliant digressions et écarts, il brise la séquentialité du texte, ce qui autorise le rapprochement avec l'art pictural: «on pourrait évoquer le genre du coq-à-l'âne pour le passage sans souci de liaison ou de cohérence d'un sujet à l'autre, d'un registre à l'autre, un peu, somme toute, sur le modèle plan et simultané du tableau» (Vasak, 2007: 23). Tous ces éléments font des Essais un texte difficile à classer, au genre mal défini et à l'allure polymorphe. L'étude des CR, dans ses rapports à la structure et à la linéarité du texte, nous permettra de voir en quoi cette impression d'ensemble est confirmée.

\subsection{Encodage numérique du corpus}

Le fichier numérique du texte provient de l'UMR ATILF (Analyse et traitement informatique de la langue française) sous le code R029. Les structures textuelles - chapitres et paragraphes - ont été encodées en XML (Extensible Markup Language) selon les recommandations de la TEI ${ }^{6}$. Le corpus TXM réalisé pour l'étude est formé des trois premiers chapitres et est appelé «DIDEROTESSAIS», l'ensemble du corpus d'étude étant désigné par «bloc» dans l'article.

5. Il peut s'agir de Grimm ou du lecteur générique.

6. Text Encoding Initiative: http://www.tei-c.org. 


\subsection{Annotation du corpus}

Le corpus a d'abord été annoté automatiquement au moment de son import dans le logiciel TXM. Il s'agit d'un étiquetage des mots en morphosyntaxe et en lemmes à l'aide du logiciel TreeTagger ${ }^{7}$ pour le français moderne.

Le corpus a ensuite fait l'objet d'une annotation manuelle en CR dans le cadre du projet DEMOCRAT ${ }^{8}$. Cette annotation se base sur la définition traditionnelle de la chaîne: font partie d'une CR toutes les expressions coréférentes d'un texte. Les limites d'unités structurelles n'entraînent pas de rupture de chaîne, ce qui nous permettra d'étudier de manière à la fois distincte et corrélée la structure textuelle et l'évolution des chaînes au fil du texte.

Les maillons des CR sont annotés comme mentions. Les mentions sont chacune associées à un référent et regroupées dans une même CR lorsqu'elles ont le même référent. La seule restriction à la constitution d'une chaine est que le nombre de mentions coréférentes doit être supérieur à deux. En deçà de ce seuil, on distingue les singletons (mentions isolées) et les paires (successions de deux mentions, que les «notions d'anaphore et de coréférence suffisent amplement à décrire», Schnedecker et Landragin, 2014: 4). Les singletons et les paires seront parfois pris en compte dans nos analyses, mais uniquement par comparaison avec les chaînes proprement dites.

Une annotation complémentaire des mentions est ensuite réalisée en mode semi-automatique, en deux passes. Il s'agit d'une annotation en catégories des mentions selon qu'elles réfèrent par pronoms personnels, déterminants possessifs, syntagmes nominaux définis, indéfinis, etc. Une macro attribue automatiquement une catégorie aux mentions (sur la base des étiquettes morphosyntaxiques des mots qui composent chaque mention), qu'un annotateur humain vérifie dans un second temps. Les annotations en références et en catégories sont ensuite vérifiées par un second annotateur ${ }^{9}$.

Lorsque l'annotation en mentions est terminée, on réalise l'annotation en $\mathrm{CR}$ en rassemblant automatiquement dans un même ensemble (un schéma dans le modèle URS) les mentions qui partagent le même référent. Le corpus TXM annoté résultant fait partie du corpus DEMOCRAT sous le nom de fichier «DIDEROTESSAIS.txm».

Une annotation complémentaire est ensuite réalisée pour les besoins de la présente étude par le calcul de la propriété «accessibilité» des mentions et des propriétés «cibles»et «empan» des CR.

7. Voir: https://www.cis.uni-muenchen.de/-schmid/tools/TreeTagger/.

8. Les principes d'annotation sont précisés dans le manuel d'annotation DEMOCRAT: http://www.lattice. cnrs.fr/democrat/files/ANR-15-CE38-0008-DEMOCRAT_livrable_methodo.pdf.

9. Tout le travail d'annotation et de vérification a été réalisé à l'aide des nouvelles interfaces d'annotation interactive offertes par l'extension «Annotation URS» de TXM développée dans le cadre du projet ANR DEMOCRAT. 


\subsection{L'extension «Annotation URS» de TXM: \\ un outil pour annoter, vérifier et exploiter}

L'extension «Annotation URS» de TXM ajoute des fonctionnalités et des interfaces pour l'annotation dynamique de modèles d'annotation URS (Unité-Relation-Schéma) pour tous les corpus gérés par TXM. Le modèle URS a été défini et implémenté à l'origine dans le logiciel Glozz (Widlöcher et Mathet, 2009). Il a également été implémenté dans le logiciel Analec (Landragin et al., 2012).

L'extension TXM reprend l'implémentation du logiciel Analec en la rendant compatible avec l'environnement de la plateforme TXM (architecture des corpus textuels, outils d'exploitation, interface utilisateur intégrée, outils d'import et d'export de textes, d'annotations et de résultats) tout en développant de nouvelles fonctionnalités basées sur ce modèle, comme par exemple l'interrogation croisée des structures textuelles (issues de l'encodage TEI à l'import) et des annotations URS des CR pour les extractions et les décomptes de cette étude.

L'extension permet d'annoter interactivement les unités au sein des éditions de texte de TXM, d'enrichir les annotations d'unités, de schémas et de relations par commandes ou par macros, de vérifier leur cohérence et de procéder à diverses extractions pour affichage ou décomptes.

\section{Chaînes de référence et homogénéité des unités structurelles}

La double annotation des Essais en CR et en unités structurelles permet de se faire une idée assez précise des caractéristiques des CR de chaque unité. Nos observations porteront avant tout sur les chapitres et quelques sondages réalisés sur les paragraphes seront évoqués en fin de section.

Les trois chapitres étudiés sont de taille comparable (autour de 3000 tokens, ponctuation comprise). Les mesures qui suivent sont réalisées sur chacun d'entre eux et les CR se définissent relativement à cette unité: une CR du chapitre 1 est une chaîne qui a au moins trois mentions dans le chapitre 1, indépendamment du fait qu'elle soit présente ou non dans une autre partie du texte.

Les premières mesures ne détaillent pas les chaines mais les analysent en bloc à l'intérieur de chaque chapitre. Les mesures suivantes permettront de distinguer des types de chaînes dans les chapitres.

\subsection{Caractéristiques générales des chaînes des chapitres}

On s'intéresse tout d'abord à la densité référentielle ou proportion d'expressions référentielles relativement à tous les mots du chapitre, que ces expressions soient ou non intégrées à des chaînes (tableau 1).

Les chiffres sont proches pour les trois chapitres et pour le bloc dans son ensemble. Où qu'on se situe dans le texte, un peu moins d'un tiers des mots sont des expressions référentielles. Cette donnée peut être complétée par le calcul de 
la couverture du texte par les chaînes. Pour évaluer cette couverture, on commence par établir le rapport entre le nombre de mots des chaînes et le nombre de mots du texte (tableau 2).

On compare ensuite le nombre de maillons des chaînes au nombre d'expressions référentielles (ou mentions) des chapitres (tableau 3).

\begin{tabular}{|l|l|}
\hline Unité & Densité référentielle \\
\hline Chapitre 1 & $27,04 \%$ \\
\hline Chapitre 2 & $29,66 \%$ \\
\hline Chapitre 3 & $29,39 \%$ \\
\hline Bloc & $28,64 \%$ \\
\hline
\end{tabular}

Tableau 1 - Densité référentielle des chapitres

\begin{tabular}{|l|l|}
\hline Unité & Densité des chaînes (en nombre de mots) \\
\hline Chapitre 1 & $12,66 \%$ \\
\hline Chapitre 2 & $12,89 \%$ \\
\hline Chapitre 3 & $9,57 \%$ \\
\hline Bloc & $12,62 \%$ \\
\hline
\end{tabular}

Tableau 2 - Densité en chaînes des chapitres (en nombre de mots)

\begin{tabular}{|l|l|l|l|}
\hline Unité & Maillons de chaînes & Mentions & Densité \\
\hline Chapitre 1 & 381 & 814 & $46,8 \%$ \\
\hline Chapitre 2 & 379 & 872 & $43,5 \%$ \\
\hline Chapitre 3 & 270 & 829 & $32,6 \%$ \\
\hline Bloc $^{10}$ & 1108 & 2515 & $44 \%$ \\
\hline
\end{tabular}

Tableau 3 - Densité en chaînes des chapitres (en nombre de mentions)

10. La somme des mentions des CR de chaque chapitre n'est pas strictement égale au nombre de mentions des CR du bloc dans la mesure où $78 \mathrm{CR}$ ont moins de 3 mentions dans un chapitre tout en ayant au moins 3 mentions dans le bloc. 


\begin{tabular}{|l|l|l|l|l|}
\hline Unités & Singletons & Paires & CR & Total \\
\hline Chapitre 1 & 325 & 54 & 56 & 435 \\
\hline Chapitre 2 & 355 & 69 & 51 & 475 \\
\hline Chapitre 3 & 389 & 85 & 48 & 522 \\
\hline Bloc & 1069 & 208 & 155 & 1432 \\
\hline
\end{tabular}

Tableau 4 - Nombre de singletons, de paires et de CR des chapitres ${ }^{11}$

Les tableaux 2 et 3 signalent tous deux que les CR occupent une part plus faible du chapitre 3. Le tableau 4, qui détaille le nombre de singletons, de paires et de CR, permet de comprendre que cette singularité tient au fait que le nombre de CR est moins élevé dans ce chapitre. Le déficit en $\mathrm{CR}$ y est compensé par un nombre plus important de singletons et de paires. Il y a donc au total beaucoup de référents dans le chapitre 3 (522 au total), mais un grand nombre d'entre eux disparaît très vite, avant même de former une chaîne. Il peut arriver qu'un référent ne soit pas mentionné plus de deux fois dans le chapitre 3 mais qu'il l'ait été suffisamment dans les chapitres précédents pour construire une CR à l'échelle du bloc. Ces cas sont rares (voir note 11) et ne changent pas la situation d'ensemble.

La section suivante apportera une indication supplémentaire: les chaînes du chapitre 3 sont moins longues que celles des deux autres chapitres.

\subsection{Types de chaînes des chapitres}

La mesure de la longueur des chaînes en nombre de mentions permet d'établir une première typologie. On peut ainsi comparer les chaînes courtes (3 et 4 mentions), intermédiaires (entre 5 et 10 mentions) et longues (plus de 10 mentions) à l'intérieur des chapitres (tableau 5).

\begin{tabular}{|l|l|l|l|l|l|l|}
\hline \multirow{2}{*}{ Unité } & \multicolumn{3}{|c|}{ CR courtes } & CR intermédiaires & CR longues & Total \\
\cline { 2 - 7 } & 3 maillons & 4 maillons & Total & 5 à 10 maillons & $\begin{array}{l}\text { Plus de } \\
10 \text { maillons }\end{array}$ & \\
\hline Chapitre 1 & 22 & 9 & 31 & 20 & 5 & 56 \\
\hline Chapitre 2 & 24 & 7 & 31 & 10 & 10 & 51 \\
\hline Chapitre 3 & 21 & 10 & 31 & 12 & 5 & 48 \\
\hline Bloc & 67 & 26 & 93 & 42 & 20 & 155 \\
\hline
\end{tabular}

Tableau 5 - Longueur des CR des chapitres

11. Il faut signaler en outre $5 \mathrm{CR}$ qui ont moins de 3 mentions dans chaque chapitre mais qui en ont plus de 3 dans le bloc et 15 paires dont les 2 mentions ne sont pas dans le même chapitre. 


\begin{tabular}{|l|l|}
\hline Unité & Chaîne la plus longue \\
\hline Chapitre 1 & 42 mentions \\
\hline Chapitre 2 & 50 mentions \\
\hline Chapitre 3 & 29 mentions \\
\hline
\end{tabular}

Tableau 6 - Taille de la CR la plus longue des chapitres (en mentions)

\begin{tabular}{|l|l|l|l|l|}
\hline Unité & $\begin{array}{l}\text { CR de } \\
\text { paragraphe }\end{array}$ & CR de chapitre & CR de bloc & Total \\
\hline Chapitre 1 & 28 & 21 & 7 & 56 \\
\hline Chapitre 2 & 28 & 4 & 19 & 51 \\
\hline Chapitre 3 & 28 & 10 & 10 & 48 \\
\hline
\end{tabular}

Tableau 7 - Empan des CR des chapitres

Les chaînes courtes sont en nombre très constant. Le chapitre 1 est plus fourni en CR intermédiaires, le chapitre 2 en CR longues. Le chapitre 3 se distingue en ce qu'il n'a ni beaucoup de CR intermédiaires, ni beaucoup de CR longues. Il présente donc peu de CR au total, beaucoup de singletons et de paires et des CR moins longues. L'examen de la taille de la CR la plus longue des chapitres confirme cette dernière particularité (tableau 6).

Le deuxième critère qui permet de définir une typologie des chaînes est l'empan des chaînes. On dénombre trois types de chaines: les CR qui ne dépassent pas les limites d'un paragraphe, celles qui se limitent au chapitre et celles qui vont au-delà (tableau 7).

Les trois types de CR du tableau 7 ne se recouvrent jamais. Dans le chapitre 1 par exemple, on dénombre $28 \mathrm{CR}$ dont toutes les mentions sont dans un seul paragraphe, $21 \mathrm{CR}$ qui courent toujours sur plus d'un paragraphe mais sans dépasser les limites du chapitre, et seulement 7 CR qui sont instanciées au moins une fois dans un autre chapitre du bloc.

Le nombre de CR qui se limitent à un seul paragraphe reste remarquablement stable quel que soit le chapitre et ce nombre est toujours plus élevé que les autres. Pour le reste, chaque chapitre a un profil particulier. Les CR du premier chapitre restent pour la plupart cantonnées dans cette unité. La situation est inversée dans le chapitre deux et les deux types de CR sont parfaitement équilibrés dans le chapitre 3. La fréquence des CR dépassant le chapitre 2 n'est pas très surprenante, dans la mesure où les titres des chapitres 2 et 3 («Mes petites idées sur la couleur» et «Tout ce que j’ai compris de ma vie du clair-obscur») laissent penser que leurs 
thématiques sont liées. On pouvait donc s'attendre à ce qu'un certain nombre de référents présents dans le chapitre 2 soient repris dans le suivant. La position intermédiaire de ce chapitre peut également introduire un biais dans l'analyse, puisqu'il a plus de chances d'avoir des chaînes qui se prolongent soit dans le chapitre qui précède soit dans celui qui suit.

\subsection{Bilan sur l'homogénéité des unités structurelles}

L'analyse comparée des $\mathrm{CR}$ des trois chapitres des Essais révèle une situation contrastée. Si la densité référentielle est assez stable au fil du texte, la couverture des chapitres par les chaînes et les caractéristiques de ces chaînes sont plus hétérogènes.

Le chapitre 1 se distingue par le fait que la plupart de ses référents ne sont pas réinstanciés au-delà du chapitre. On note toutefois un nombre important de $\mathrm{CR}$ qui dépassent les limites d'un paragraphe et se poursuivent dans le chapitre, ce qui est peut-être à mettre en relation avec le nombre important de CR de longueur intermédiaire (entre 5 et 10 mentions).

Le chapitre 2 comporte beaucoup de CR qui sont présentes ailleurs dans le bloc. C'est également dans ce chapitre qu'on rencontre le nombre le plus important de CR longues (dans la limite du chapitre).

Le chapitre 3 se distingue par la rapidité avec laquelle ses référents disparaissent: il comporte moins de CR, des CR qui sont globalement moins longues et qui occupent une moindre part du texte.

Les trois chapitres partagent une même particularité: la moitié au moins de leurs chaînes se cantonne à un seul paragraphe. Ce résultat n'a rien d'étonnant. De même que les chaînes courtes sont toujours les plus nombreuses (dans le texte, dans le chapitre, dans le paragraphe), de même, une grande partie des chaînes a un empan très local. Il y a donc dans le texte - et ce constat vaut probablement pour tous les textes - un grand nombre de référents éphémères dont les mentions sont peu nombreuses et très rapprochées (dans le même paragraphe).

Cinq paragraphes de taille comparable (autour de 300 mots) extraits des trois chapitres ont été analysés les uns indépendamment des autres selon le même protocole. Les résultats de cette analyse révèlent de grandes disparités. La densité référentielle, la couverture et la densité en chaînes, la longueur et l'empan des chaînes varient dans des proportions importantes, ce qui montre qu'à l'intérieur d'un texte comme les Essais l'étude des CR peut donner des résultats assez différents selon les paragraphes qu'on examine. Notre étude permet en revanche de repérer deux constantes: le nombre de CR varie peu dans des unités de taille comparable (4 ou 6 chaînes pour 300 mots) et lorsque ce nombre est plus élevé ( 6 chaînes) ce sont en réalité les chaînes courtes qui augmentent. Ce constat renforce le poids des $\mathrm{CR}$ courtes dans la cartographie générale.

L'étude confirme ainsi le rôle micro-textuel des CR. La plupart des chaînes sont très courtes, plus de la moitié d'entre elles ne dépassent pas le niveau méso-textuel 
du paragraphe et leur nombre dans le paragraphe semble très dépendant de sa taille. Le fait que l'auteur des Essais organise ses unités de paragraphe autour d'un petit nombre de CR pose par ailleurs la question de leur relation à l'unité thématique. Nous tenterons de préciser ce lien dans la section qui suit.

\section{Chaînes de référence et thèmes des unités structurelles}

Le thème est entendu ici au sens «classique» de thème de discours (Marandin, 1988:

68) et défini grâce à la relation d'«à propos»: c'est ce dont le discours "parle», ce à propos de quoi il dit quelque chose. Nous sommes conscients qu'il s'agit là d'une définition parmi d'autres, qui tend à calquer de manière un peu simpliste la notion de thème de discours sur celle de thème de phrase en supposant que le thème est porté par un constituant de l'énoncé. Mais parce qu'elle établit un lien direct entre le thème et les référents mentionnés à l'intérieur des chaînes, cette définition a le mérite d'offrir un point de départ qui parait opératoire (on verra que les moyens mis en œuvre pour l'étude deviennent vite complexes, en particulier lorsqu'il s'agit de traiter une grande masse de données).

Pour réaliser cette étude, nous nous inspirons du modèle de Givón (1983) et de la manière dont il articule les thèmes et les référents discursifs (qu'il appelle «topiques») en les situant sur des niveaux d'organisation différents. Givón distingue en réalité non pas deux mais trois niveaux qui s'emboîtent les uns dans les autres et qui s'organisent autour de trois types de continuité/discontinuité discursive: la continuité thématique («thematic continuity») > la continuité d'action («action continuity») > la continuité topicale («topics/participants continuity»). L'intrication de ces trois niveaux explique qu'il y ait une corrélation assez forte entre la continuité thématique et la continuité topicale: ce dont traite une unité thématique, que Givón appelle «paragraphe thématique» (indépendamment du découpage graphique), ce sont généralement un ou plusieurs référents (ou topiques) proéminents. Ces référents les plus centraux sont le plus souvent les topiques les plus continus de cette unité. Il y a donc a priori une association étroite entre les référents les plus continus, les plus centraux/proéminents et ceux qui définissent le thème du discours:

Within the thematic paragraph it is most common for one topic to be the continuity marker, the leitmotif, so that it is the participant most crucially involved in the action sequence running through the paragraph; it is the participant most closely associated with the higher-level "theme" of the paragraph" most likely to be coded as the primary topic - or grammatical subject - of the vast majority of sequentially-ordered clauses/sentences comprising the thematic paragraph. It is thus, obviously, the most continuous of all the topics mentioned in the various clauses in the paragraph.

(Givón, 1983: 8)

12. C'est nous qui soulignons. 
La continuité topicale pourrait ainsi permettre d'approcher ce qui fait l'unité d'un paragraphe thématique et donner un moyen d'identifier son thème. Elle se mesure notamment à travers la persistance du référent: on s'attend en effet à ce qu'un référent continu soit réinstancié un grand nombre de fois et soit récurrent dans l'unité thématique. La longueur des CR sera pour nous une manière de mesurer la persistance des référents à l'intérieur des unités structurelles. La fréquence des catégories grammaticales permettra de compléter l'analyse croisée de la continuité topicale et thématique, certaines catégories (les marques de haute accessibilité) marquant a priori la continuité maximale, d'autres au contraire une continuité plus faible (les marques d'accessibilité moyenne ou faible).

\subsection{Application du cadre d'analyse au corpus}

Dans cette section, nous envisageons les unités structurelles (bloc, chapitres et paragraphes) comme des unités thématiques. En ce sens, nous faisons l'hypothèse que l'auteur développe dans chaque unité un thème qui lui est propre et que ce thème est sensiblement différent de celui de l'unité qui précède et qui suit. Nous n'irons pas jusqu'à prétendre que chaque paragraphe procède d'un saut thématique radical mais qu'il existe d'un paragraphe à l'autre une évolution sensible. Puisque selon la même hypothèse un chapitre est aussi en soi une unité thématique, les différents paragraphes qui le composent devront à la fois participer d'un thème commun (celui du chapitre) tout en ayant une déclinaison qui leur est propre au niveau du paragraphe. Dans ce cadre, nous cherchons à savoir de manière empirique si les CR qui jalonnent ces unités structurelles sont à même d'aider à identifier, par le biais de leurs propriétés, le thème de ces unités. Nous étudions pour cela trois propriétés en particulier: (1) la longueur des chaînes (on suppose que le thème d'une unité est porté par les référents le plus souvent mentionnés, les plus persistants), (2) l'empan des chaînes (le thème serait porté par les référents qu'on ne mentionne pas ailleurs dans une autre unité) et (3) l'accessibilité des référents (le thème serait porté par les référents les plus accessibles, les plus présents en mémoire).

\subsection{Méthode : définition des thèmes et des chaînes cibles}

Le bloc que nous étudions est composé de 3 chapitres eux-mêmes divisés en 71 paragraphes au total. Si les chapitres sont de taille comparable, les paragraphes sont au contraire de taille très variable. Dans cette section, nous nous focaliserons sur les 3 paragraphes les plus longs de chaque partie (tableau 8).

\begin{tabular}{|l|l|l|l|l|l|l|l|l|l|}
\hline Chapitre & \multicolumn{3}{|c|}{1} & \multicolumn{3}{c|}{2} & \multicolumn{3}{c|}{3} \\
\hline Paragraphe & 14 & 17 & 22 & 29 & 44 & 46 & 53 & 63 & 66 \\
\hline Taille (mots) & 529 & 268 & 298 & 287 & 394 & 267 & 308 & 287 & 296 \\
\hline
\end{tabular}

Tableau 8 - Taille des paragraphes retenus pour l'étude «thématique» 
Du point de vue des thèmes des unités structurelles, la situation est à nouveau inégale. Les chapitres ont un nom donné par l'auteur, que l'on peut prendre pour thème, tandis que les paragraphes n'en ont aucun. Les titres de chapitre sont les suivants:

Chapitre 1: «Mes pensées bizarres sur le dessin»

Chapitre 2: «Mes petites idées sur la couleur»

Chapitre 3: «Tout ce que j'ai compris de ma vie du clair-obscur»

Pour établir un lien entre les chaînes et le thème des paragraphes, nous recourons à une méthode manuelle qui consiste à annoter chacun d'eux pour lui attribuer une sorte de titre et déterminer ainsi ses éléments thématiques. Cette méthode implique une certaine subjectivité du lecteur, que nous allons modérer en procédant à une double annotation. Chaque annotateur lit et propose un thème pour chaque paragraphe. Les deux annotateurs confrontent ensuite leurs propositions et s'accordent sur une description commune. En procédant ainsi, nous disposons de descriptions thématiques pour les deux niveaux de structure, le chapitre et le paragraphe. Nous concédons que ces descriptions ne sont pas de la même nature ou du moins de la même origine, puisque les premières sont fournies par l'auteur dans un certain dessein tandis que les autres sont construites par un binôme de lecteurs. Il s'agit d'une première approche d'un problème difficile (on a mentionné plus haut le flou conceptuel qui entoure en général la notion de thème de discours, voir Marandin, 1988).

Après annotation des unités structurelles, la liste des thèmes est donnée dans le tableau 9 (les guillemets signalent qu'il s'agit du titre original).

La phase ultime de la préparation des données consiste à relever parmi toutes les CR celles dont les référents sont sémantiquement proches du thème annoté dans l'unité. Pour ce faire, nous considérons chaque unité comme un sous-corpus et dressons la liste des chaînes candidates (il faut notamment qu'elles aient au moins trois mentions à l'intérieur de l'unité). Ces chaînes candidates à exprimer le thème de l'unité sont appelées chaînes cibles. On ne tient aucun compte dans cette phase des propriétés des chaînes et on se base uniquement sur des rapports sémantiques.

Dans un second temps, nous étudierons les propriétés de longueur et d'empan des chaînes cibles ainsi que l'accessibilité de leurs référents (mesurée grâce aux catégories grammaticales des mentions), en les comparant aux autres chaînes des unités.

Pour le premier chapitre, nous retiendrons les CR de l'auteur, ses pensées ou réflexions et le dessin pris au sens large (peinture, tableau, œuvre, esquisse, croquis, etc.). Parmi les 56 chaînes qui figurent dans le chapitre, nous ne retiendrons donc que 3 chaînes cibles: l'auteur ( 42 mentions), le dessin ( 4 mentions) et l'écorché (le dessin anatomique, 8 mentions).

Pour le paragraphe 14 et ses 11 chaînes, nous retiendrons «l'Académie» (7 mentions) ainsi que «les actions, les positions et les figures fausses, apprêtées et froides» (7 mentions) pour exprimer la notion de vérité. 


\begin{tabular}{|c|c|}
\hline Unité & Thème ou titre \\
\hline Chapitre 1 & «Mes pensées bizarres sur le dessin» \\
\hline Paragraphe 14 & $\begin{array}{l}\text { Le dessin académique d'après modèle empêche de peindre la nature } \\
\text { en vérité }\end{array}$ \\
\hline Paragraphe 17 & $\begin{array}{l}\text { Nécessité de peindre une action véritable plutôt qu'un modèle } \\
\text { cherchant à imiter }\end{array}$ \\
\hline Paragraphe 22 & L’école de dessin idéale \\
\hline Chapitre 2 & «Mes petites idées sur la couleur» \\
\hline Paragraphe 29 & La variété des couleurs est liée à celle des états d’âme du peintre \\
\hline Paragraphe 44 & $\begin{array}{l}\text { La palette des couleurs d'un artiste est plus limitée que celle de la } \\
\text { nature }\end{array}$ \\
\hline Paragraphe 46 & Les couleurs de la chair sont très fugaces et difficiles à fixer \\
\hline Chapitre 3 & «Tout ce que j’ai compris de ma vie du clair-obscur» \\
\hline Paragraphe 53 & $\begin{array}{l}\text { Par leur grande beauté, certains paysages d'ombre et de lumière nous } \\
\text { paraissent tels des œuvres d'art }\end{array}$ \\
\hline Paragraphe 63 & $\begin{array}{l}\text { L'éclat de la lumière influence la couleur des objets et celle de leur } \\
\text { ombre }\end{array}$ \\
\hline Paragraphe 66 & $\begin{array}{l}\text { Comme la lumière influe sur la couleur, elle joue aussi sur la teinte } \\
\text { des ombres }\end{array}$ \\
\hline
\end{tabular}

Tableau 9 - Les thèmes des unités structurelles

Le paragraphe 17 ne comporte que 3 chaînes. Les trois référents que l'auteur mentionne à plus de trois reprises dans le paragraphe sont: «l'auteur», «les deux camarades» et «les jeunes élèves». Aucun de ces référents n'exprime même en partie le thème annoncé. Ce paragraphe sera pour cette raison exclu de la suite des analyses.

Le reste des unités a été traité selon le même principe. La liste des chaînes cibles est donnée dans le tableau 10 (le nombre entre parenthèses indique la longueur, c'est-à-dire le nombre de fois que le référent est mentionné dans l'unité considérée).

On notera que chaque chapitre est relativement bien thématisé et que le thème ressort partiellement à partir des référents des chaînes : le premier porte plutôt sur le dessin académique (et pourrait sans doute avoir un autre titre que celui donné par l'auteur), le deuxième sur la couleur et le troisième sur la lumière. Chaque paragraphe reprend en général une partie du thème du chapitre et développe une ou deux chaînes particulières. Mais le lien qui existe entre les chaines cibles et le thème est plus ou moins direct selon les paragraphes (voir par exemple les paragraphes 29, 44 et 53). En effet, le thème est parfois davantage porté par des exemples variés et 
spécifiques («les vieux arbres épargnés» pour «les paysages d'ombre») et par ailleurs par des référents qui ne sont pas suffisamment répétés pour constituer des chaînes ${ }^{13}$.

Nous étudions à présent si les propriétés énoncées plus haut - longueur, empan des chaînes et accessibilité des référents - permettent ou non d'identifier le thème des unités structurelles.

\begin{tabular}{|c|c|c|}
\hline Unité & Thème ou titre & Chaînes cibles (référents) \\
\hline Chapitre 1 & $\begin{array}{l}\text { «Mes pensées bizarres sur le } \\
\text { dessin» }\end{array}$ & $\begin{array}{l}\text { l'auteur (40), l'écorché (8), le } \\
\text { dessin (4) }\end{array}$ \\
\hline Paragraphe 14 & $\begin{array}{l}\text { Le dessin académique d'après } \\
\text { modèle empêche de peindre la } \\
\text { nature en vérité }\end{array}$ & $\begin{array}{l}\text { l'Académie (7), les figures } \\
\text { fausses (7) }\end{array}$ \\
\hline Paragraphe 17 & $\begin{array}{l}\text { Nécessité de peindre une action } \\
\text { véritable plutôt qu'un modèle } \\
\text { cherchant à imiter }\end{array}$ & (aucun) \\
\hline Paragraphe 22 & L'école de dessin idéale & $\begin{array}{l}\text { l'élève de l'école de dessin (10), le } \\
\text { modèle académique (3) }\end{array}$ \\
\hline Chapitre 2 & «Mes petites idées sur la couleur» & $\begin{array}{l}\text { l'auteur (34), la couleur (26), les } \\
\text { couleurs (7) }\end{array}$ \\
\hline Paragraphe 29 & $\begin{array}{l}\text { La variété des couleurs est liée à } \\
\text { celle des états d'âme du peintre }\end{array}$ & $\begin{array}{l}\text { le même voile jaune (3), l'homme } \\
\text { qui peint (29) }\end{array}$ \\
\hline Paragraphe 44 & $\begin{array}{l}\text { La palette des couleurs d'un artiste } \\
\text { est plus limitée que celle de la nature }\end{array}$ & les couleurs (6), l'arc-en-ciel (3) \\
\hline Paragraphe 46 & $\begin{array}{l}\text { Les couleurs de la chair sont très } \\
\text { fugaces et difficiles à fixer }\end{array}$ & $\begin{array}{l}\text { le coloriste vrai (5), la chair (3), ce } \\
\text { qui achève de rendre fou le grand } \\
\text { coloriste (4) }\end{array}$ \\
\hline Chapitre 3 & $\begin{array}{l}\text { «Tout ce que j'ai compris de ma vie } \\
\text { du clair-obscur» }\end{array}$ & $\begin{array}{l}\text { l'auteur (29), le clair-obscur (3), } \\
\text { la lumière (25), les ombres (12), } \\
\text { la lumière générale (3), les } \\
\text { lumières (7) }\end{array}$ \\
\hline Paragraphe 53 & $\begin{array}{l}\text { Par leur grande beauté, certains } \\
\text { paysages d'ombre et de lumière } \\
\text { nous paraissent tels des œuvres d'art }\end{array}$ & $\begin{array}{l}\text { les vieux arbres épargnés (3), le } \\
\text { soleil (4), les rayons obliques du } \\
\text { soleil (6) }\end{array}$ \\
\hline Paragraphe 63 & $\begin{array}{l}\text { L'éclat de la lumière influence la } \\
\text { couleur des objets et celle de leur } \\
\text { ombre }\end{array}$ & $\begin{array}{l}\text { la lumière (4), la lumière générale } \\
\text { (3) }\end{array}$ \\
\hline Paragraphe 66 & $\begin{array}{l}\text { Comme la lumière influe sur la } \\
\text { couleur, elle joue aussi sur la teinte } \\
\text { des ombres }\end{array}$ & la lumière (4), les ombres (5) \\
\hline
\end{tabular}

Tableau 10 - Liste des chaines cibles par unité structurelle

13. On trouve en général 3 à 7 chaînes par paragraphe (400 mots). 


\subsection{Longueur des chaînes} les sujets), dont malheureusement une seule est une chaîne cible (l'élève de l'école de dessin). Cela lui vaut un score de précision de $1 / 3$, soit $33 \%$. Elle ne rappelle qu'une chaîne cible sur les deux à trouver (elle manque le modèle académique).

La méthode propose donc 3 chaînes longues (l'élève de l'école de dessin, l'auteur,

Voici donc comment $[j e]_{\mathrm{a}}$ désirerais qu'une école de dessin fût conduite. Lorsque [l'élève $]_{\mathrm{e}}$ sait dessiner facilement d'après l'estampe et la bosse, $[j e]_{\mathrm{a}}[l \boldsymbol{l}]_{\mathrm{e}}$ tiens pendant deux ans devant [le modèle académique de l'homme et de la femme $]_{\mathrm{m}}$. Puis $[j e]_{\mathrm{a}}$ $[\text { lui }]_{\mathrm{e}}$ expose des enfants, des adultes, des hommes faits, des vieillards, [des sujets $]_{\mathrm{s}}$ de tout âge, de tout sexe, pris dans toutes les conditions de la société, toutes sortes de natures, en un mot. [Les sujets $]_{\mathrm{s}}$ se présenteront en foule à la porte de $[\text { mon }]_{\mathrm{a}}$ académie, si $[j e]_{\mathrm{a}}[l e s]_{\mathrm{s}}$ paie bien; si $[j e]_{\mathrm{a}}$ suis dans un pays d'esclaves, $[j e]_{\mathrm{a}}[l e s]_{\mathrm{s}} \mathrm{y}$ ferai venir. Dans [ces différents modèles $]_{\mathrm{s}}$ le professeur aura soin de $[l u i]_{\mathrm{e}}$ faire remarquer les accidents que les fonctions journalières, la manière de vivre, la condition et l'âge ont introduits dans les formes. $\left[[M o n]_{\mathrm{a}} \text { élève }\right]_{\mathrm{e}}$ ne reverra plus [le modèle académique $]_{\mathrm{m}}$ qu'une fois tous les quinze jours; et le professeur abandonnera [au modèle $]_{\mathrm{m}}$ le soin de se poser [lui-même $]_{\mathrm{m}}$. Après la séance de dessin un habile anatomiste expliquera à $\left[[m o n]_{\mathrm{a}} \text { élève }\right]_{\mathrm{e}}$ l'écorché, et $[l u i]_{\mathrm{e}}$ fera l'application de ses leçons sur le nu animé et vivant; et $[i l]_{\mathrm{e}}$ ne dessinera d'après l'écorché que douze fois au plus dans une année. C'en sera assez pour qu' $[i]_{\mathrm{e}}$ sente que les chairs sur les os et les chairs non appuyées ne se dessinent pas de la même manière, qu'ici le trait est rond, là comme anguleux; et que s' $[i]_{\mathrm{e}}$ néglige ces finesses, le tout aura l'air d'une vessie soufflée ou d'une balle de coton.

On note qu'il y a dans ce paragraphe deux chaines cibles: celle de l'élève de l'école de dessin, indexée par la lettre $e$, et celle du modèle académique, indexée par $m$. L'une est longue (l'élève, 10 mentions), l'autre est courte (le modèle, 4 mentions). Il y a dans le paragraphe deux autres chaînes longues: la chaîne de l'auteur (9 mentions, indexée par $a$ ), qui comporte une majorité de pronoms déictiques dont on peut se demander s'ils forment réellement une chaîne, et la chaîne des sujets (5 mentions, indexée par $s$ ). 
Son taux de rappel est de $1 / 2$, soit $50 \%$. La longueur des chaînes n'est donc pas un critère suffisamment précis pour cibler le thème de ce paragraphe.

\subsection{Empan des chaînes}

L'hypothèse à tester est que la spécificité d'un thème local est portée principalement par des chaînes qui ne sont pas développées ailleurs dans le texte, dans une autre unité de même niveau. Nous attribuons donc une propriété particulière à chaque chaîne, son empan, qui qualifie la chaîne selon la position des mentions dans les unités. Nous aurons ainsi des chaines de paragraphe (dont les limites ne dépassent pas le paragraphe), de chapitre ou de bloc.

Comme précédemment, nous illustrons l'application de ce critère grâce à l'exemple du paragraphe 22. Nous mettons en italique les chaînes spécifiques à ce paragraphe. Nous indiquerons toujours en gras les chaînes cibles.

Voici donc comment je désirerais qu'une école de dessin fût conduite. Lorsque [l'élève $]_{\mathrm{e}}$ sait dessiner facilement d'après l'estampe et la bosse, je $[\mathbf{l e}]_{\mathrm{e}}$ tiens pendant deux ans devant [le modèle académique de l'homme et de la femme $]_{\mathrm{m}}$. Puis je [lui $]_{e}$ expose des enfants, des adultes, des hommes faits, des vieillards, [des sujets $]_{s}$ de tout âge, de tout sexe, pris dans toutes les conditions de la société, toutes sortes de natures, en un mot. [Les sujett] $]_{s}$ se présenteront en foule à la porte de mon académie, si je $[l e s]_{s}$ paie bien; si je suis dans un pays d'esclaves, je $[l e s]_{\mathrm{s}}$ y ferai venir. Dans [ces différents modèles $]_{\mathrm{s}}$ le professeur aura soin de [lui $]_{\mathrm{e}}$ faire remarquer les accidents que les fonctions journalières, la manière de vivre, la condition et l'âge ont introduits dans les formes. [Mon élève $]_{\mathrm{e}}$ ne reverra plus [le modèle académique $]_{\mathrm{m}}$ qu'une fois tous les quinze jours; et le professeur abandonnera [au modèle $]_{\mathrm{m}}$ le soin de se poser $[\text { [ui-même }]_{\mathrm{m}}$. Après la séance de dessin un habile anatomiste expliquera à [mon élève $]_{\mathrm{e}}$ l'écorché, et [luii $]_{\mathrm{e}}$ fera l'application de ses leçons sur le nu animé et vivant; et $[\mathbf{i l}]_{\mathrm{e}}$ ne dessinera d'après l'écorché que douze fois au plus dans une année. C'en sera assez pour qu' $[\mathbf{i l}]_{\mathrm{e}}$ sente que les chairs sur les os et les chairs non appuyées ne se dessinent pas de la même manière, qu'ici le trait est rond, là comme anguleux; et que s'[il] e néglige ces finesses, le tout aura l'air d'une vessie soufflée ou d'une balle de coton.

Le paragraphe comporte toujours les mêmes deux chaînes cibles: celle de l'élève de l'école de dessin et celle du modèle académique. L'une est spécifique à ce paragraphe (le modèle académique), l'autre ne l'est pas (l'élève de l'école de dessin est aussi mentionné dans d'autres paragraphes). On détecte une autre chaîne spécifique à ce paragraphe, celle des sujets. La méthode identifie donc deux chaines spécifiques à ce paragraphe (le modèle académique et les sujets), dont une seule chaîne candidate à l'explicitation du thème. Son score de précision n'est que de $50 \%$. On note que la chaine cible repérée par cette méthode (le modèle académique) n'avait pas été détectée par la méthode précédente, parce que trop courte. La chaine des sujets, non candidate d'après nous à la définition du thème du paragraphe, se distingue pourtant par sa longueur et par le fait qu'elle est spécifique à ce paragraphe. 


\subsection{Accessibilité des référents des chaînes}

L'hypothèse que nous souhaitons tester ici est que le thème, toujours fortement présent en mémoire, apparait plutôt sous la forme d'une expression qui marque la haute accessibilité du référent (pronom personnel anaphorique, pronom relatif, déterminant possessif et sujet zéro dans le cas de l'ellipse du sujet). Nous repérons donc les chaînes qui ont un fort taux de mentions de ce type.

Comme précédemment, nous illustrons l'exploitation du troisième critère à l'aide du paragraphe 22. Nous mettons en italique les chaînes qui ont un fort taux d'expressions référentielles marquant la haute accessibilité (c'est-à-dire au moins $50 \%$ ). Nous indiquons toujours en gras les mentions des chaînes cibles.

Voici donc comment $[j e]_{a}$ désirerais qu'une école de dessin fût conduite. Lorsque $[\text { l'élève }]_{\mathrm{e}}$ sait dessiner facilement d'après l'estampe et la bosse, $[j e]_{\mathrm{a}}[l e]_{\mathrm{e}}$ tiens pendant deux ans devant [le modèle académique de l'homme et de la femme $]_{\mathrm{m}}$. Puis $[j]_{\mathrm{a}}$ $[l u i]_{\mathrm{e}}$ expose des enfants, des adultes, des hommes faits, des vieillards, des sujets de tout âge, de tout sexe, pris dans toutes les conditions de la société, toutes sortes de natures, en un mot. Les sujets se présenteront en foule à la porte de $[\mathrm{mon}]_{\mathrm{a}}$ académie, si $[j e]_{a}$ les paie bien; si $[j e]_{a}$ suis dans un pays d'esclaves, $[j e]_{a}$ les y ferai venir. Dans ces différents modèles le professeur aura soin de $[h i]_{\mathrm{e}}$ faire remarquer les accidents que les fonctions journalières, la manière de vivre, la condition et l'âge ont introduits dans les formes. $\left[[\mathrm{Mon}]_{\mathrm{a}} \text { élève }\right]_{\mathrm{e}}$ ne reverra plus [le modèle académique $]_{\mathrm{m}}$ qu'une fois tous les quinze jours; et le professeur abandonnera [au modèle $]_{\mathrm{m}}$ le soin de se poser [lui-même $]_{\mathrm{m}}$. Après la séance de dessin un habile anatomiste expliquera à $\left[[\mathrm{mon}]_{\mathrm{a}} \text { élève }\right]_{\mathrm{e}}$ l'écorché, et $[\boldsymbol{l u i}]_{\mathrm{e}}$ fera l'application de ses leçons sur le nu animé et vivant; et $\left[i l_{\mathrm{e}}\right.$ ne dessinera d'après l'écorché que douze fois au plus dans une année. C'en sera assez pour qu' $\left[i l_{\mathrm{e}}\right.$ sente que les chairs sur les os et les chairs non appuyées ne se dessinent pas de la même manière, qu'ici le trait est rond, là comme anguleux; et que s' $[i]_{\mathrm{e}}$ néglige ces finesses, le tout aura l'air d'une vessie soufflée ou d'une balle de coton.

Les deux chaînes cibles sont toujours l'élève de l'école de dessin et le modèle académique. L'une contient une forte proportion de marques de haute accessibilité (l'élève de l'école de dessin, 70 \%), l'autre non (33\%). Une seule autre chaîne se caractérise par son taux élevé de marques de haute accessibilité : il s'agit de la chaîne de l'auteur, sur laquelle on peut émettre de nouvelles réserves étant donné ses fortes particularités (présence exclusive de pronoms personnels de première personne et de possessifs). La méthode identifie donc deux chaînes (l'élève de l'école de dessin et l'auteur), dont une seule participe à la définition du thème. Son score de précision n'est que de $50 \%$.

\subsection{Synthèse des résultats sur les chapitres et les paragraphes}

Après avoir exposé la mise en œuvre des trois méthodes, nous présentons ci-dessous deux tableaux de synthèse, l'un traitant de l'identification du thème des chapitres, l'autre du thème des paragraphes. S'agissant d'un problème d'identification d'une propriété, nous nous intéressons au score de précision des méthodes, qui mesure 
a posteriori la pertinence des chaînes proposées ${ }^{14}$. Pour chaque unité (chapitre ou paragraphe), nous indiquons combien de chaînes cibles étaient à trouver, combien de chaînes ont été proposées par chaque méthode et combien étaient correctes. Le rapport entre ces deux derniers chiffres nous donne la précision.

À la lecture du tableau 11, nous constatons qu'aucune des trois méthodes n'est en mesure d'identifier avec précision (ou fiabilité) les référents en rapport avec le thème des chapitres. Sachant qu'il y a en moyenne 4 chaînes cibles à trouver, on pourrait considérer qu'une bonne méthode obtiendrait un score moyen de 75 à 80 \% (3 chaînes correctes sur 4 proposées ou 4 chaînes correctes sur 5 proposées).

La méthode qui s'appuie sur l'empan des chaînes (c'est-à-dire sur la spécificité des référents à un chapitre donné) échoue complètement par rapport au thème du chapitre 2. La méthode qui s'en sort le mieux est celle qui s'appuie sur la propriété des longueurs des chaînes. En retenant les référents qui apparaissent au moins dix fois dans le chapitre courant, elle propose entre 4 à 5 référents, dont la moitié relève du thème du chapitre.

\begin{tabular}{|l|l|l|l|l|}
\hline Chapitre & Chaînes cibles & Longueur & Empan & Accessibilité \\
\hline 1 & 3 & $1 / 5(20 \%)$ & $1 / 6(17 \%)$ & $<1 / 5(20 \%)$ \\
\hline 2 & 3 & $2 / 4(50 \%)$ & $0 / 4(0 \%)$ & $2 / 3(67 \%)$ \\
\hline 3 & 6 & $3 / 5(60 \%)$ & $4 / 10(40 \%)$ & $<2 / 11(18 \%)$ \\
\hline Moyenne & 4 & $43 \%$ & $19 \%$ & $<35 \%$ \\
\hline
\end{tabular}

Tableau 11 - Précision des trois méthodes quant à l'identification des thèmes des chapitres

\begin{tabular}{|l|l|l|l|l|}
\hline Paragraphe & Chaînes cibles & Longueur & Empan & Accessibilité \\
\hline 14 & 2 & $2 / 5$ & $1 / 3$ & $1 / 5$ \\
\hline 22 & 2 & $1 / 3$ & $1 / 2$ & $1 / 2$ \\
\hline 29 & 2 & $1 / 1$ & $1 / 1$ & $2 / 2$ \\
\hline 44 & 2 & $1 / 3$ & $1 / 2$ & $1 / 4$ \\
\hline 46 & 3 & $1 / 2$ & $1 / 1$ & $2 / 3$ \\
\hline 53 & 3 & $1 / 1$ & $2 / 2$ & $1 / 1$ \\
\hline 63 & 2 & $0 / 1$ & $1 / 2$ & $1 / 3$ \\
\hline 66 & 2 & $1 / 2$ & $0 / 2$ & $0 / 3$ \\
\hline Moyenne & 2,25 & $51 \%$ & $60 \%$ & $49 \%$ \\
\hline
\end{tabular}

Tableau 12 - Précision des trois méthodes quant à l'identification des thèmes des paragraphes

14. Les scores en rappel sont moyens et varient peu selon les méthodes: $40 \%$ sur le thème des chapitres; $45 \%$ sur le thème des paragraphes. 
Pour le problème de l'identification du thème des paragraphes (tableau 12), il faut identifier en moyenne 2,25 chaînes cibles dans chaque paragraphe. Pour obtenir un bon score, il ne faudrait faire qu'une erreur sur trois chaînes proposées, soit un score de $66 \%$. D'après ce tableau, nous constatons que chacune des trois méthodes progresse. Elles parviennent même à atteindre le seuil des $50 \%$ avec $60 \%$ pour la méthode de l'empan. Il semblerait donc que la relation entre $\mathrm{CR}$ et thème soit plus intime au niveau des paragraphes, à une échelle plus locale, qu'au niveau plus macroscopique du chapitre.

On constate également que dans le cas des paragraphes les trois méthodes connaissent des taux de réussite relativement comparables. Chacune d'entre elles connaît une situation d'échec, mais aussi des situations de succès relatif. Contrairement à ce qui se produit avec les chapitres, les méthodes de l'empan et de l'accessibilité sont en mesure de faire des propositions pertinentes. La méthode de l'empan est d'ailleurs la plus efficace et avoisine le seuil recherché des $66 \%$, alors qu'elle était la moins précise pour l'identification du thème des chapitres. Selon cette méthode, l'unité thématique du paragraphe serait plus particulièrement liée à la rupture qui s'établit entre cette unité et les unités voisines.

\subsection{Bilan sur les thèmes des unités structurelles}

Les résultats exposés ci-dessus démontrent que le rapport entre les $\mathrm{CR}$ et le thème des unités de discours est complexe et délicat à mettre en évidence. Chacune des trois méthodes explorées, certes simplistes (mais c'est une première approche), peine à fournir avec précision et satisfaction des référents en lien avec le thème. L'étude apporte néanmoins quelques éclairages intéressants.

L'efficacité des méthodes est variable selon qu'on traite d'une unité large comme le chapitre ou plus étroite comme le paragraphe (même si nous avons sélectionné les paragraphes les plus longs du texte). Dans le cas des chapitres, le thème semble davantage porté par le maintien répété de certains référents tout au long de l'unité, d'où un meilleur score du critère de la longueur des chaînes. Dans le cas des paragraphes, c'est plutôt le caractère discriminant, spécifique, des référents qui semble jouer, comme si le thème d'un paragraphe devait se définir par opposition aux paragraphes qui l'entourent.

On notera que le critère de longueur est sans doute dépendant de la longueur du chapitre lui-même ou de sa densité référentielle. Le seuil à partir duquel on détermine qu'une chaîne est longue doit donc être apprécié relativement à d'autres propriétés de l'unité. En revanche, le critère de l'empan, qui s'appuie uniquement sur la présence ou l'absence des référents dans le restant du texte, est indépendant de la taille des unités considérées.

On remarquera enfin - au moins pour le paragraphe 22 - que les chaînes proposées par les trois méthodes sont sensiblement les mêmes. Il serait sans doute intéressant d'étudier qualitativement dans quelle mesure ces méthodes se recoupent et d'envisager le cas échéant si une certaine combinaison de celles-ci n'apporterait pas un gain significatif. 


\section{Discussion et perspectives} déterminantes. méthodologiques.
Nous avons mené une large étude sur les propriétés des CR en relation avec les unités structurelles imbriquées des Essais sur la peinture de Diderot. Nous nous sommes en particulier intéressés aux deux premiers niveaux de structure (chapitres et paragraphes) et aux propriétés de longueur, d'empan et à l'accessibilité des référents. Après un premier travail d'analyse sur la distribution des mentions dans ces structures, nous avons conduit une seconde étude sur la question du rapport entre $\mathrm{CR}$ et thème des unités structurelles. Pour ce faire, nous nous sommes appuyés tantôt sur les titres fournis par l'auteur (pour les chapitres), tantôt sur des thèmes produits par une annotation manuelle (pour les paragraphes). D'un point de vue méthodologique, il conviendrait d'homogénéiser l'approche par une annotation systématique des thèmes de toutes les unités. Cette étude multi-niveaux a néanmoins pu être réalisée grâce à l'outillage très précis offert par l'extension «Annotation URS» de TXM et aux ressources produites par le projet DEMOCRAT. Les facilités apportées par cet environnement pour définir les sous-corpus par chapitre et par paragraphe, pour croiser ainsi les propriétés de chaînes et les contraintes structurelles ont été

Il ressort de cette recherche que les CR sont pour l'essentiel cantonnées à une dimension très locale: une majorité d'entre elles ne dépasse pas les limites du paragraphe et toutes les unités structurelles des Essais comportent une forte proportion de chaînes très courtes. Pour le reste, les paragraphes se caractérisent par des variations importantes (du point de vue de la densité des chaînes, etc.) et les chapitres sont plus homogènes. Selon l'échelle à laquelle on mène l'analyse (niveau de la macro-structure ou niveau méso), l'étude des propriétés des chaînes donne ainsi des résultats bien différents, ce qui montre la difficulté à dégager des tendances stables et la complexité des rapports entre structures et chaînes.

La méthode mise en œuvre pour dégager les thèmes des unités structurelles à partir des chaînes et de leurs propriétés a montré des limites importantes, en particulier pour les unités de niveau supérieur. Notre définition du thème ainsi que le postulat selon lequel il est porté par les référents du texte peuvent naturellement être mis en cause. L'étude montre aussi que le modèle de Givón, sur lequel nous nous sommes basés, ne donne pas de résultats très satisfaisants sur les Essais. Il est possible, sinon probable, que ce modèle fonctionne mieux sur des textes narratifs, moins «digressifs», et centrés de manière constante sur quelques référents de discours. On peut aussi supposer qu'il est plus adapté à la progression à thème constant qu'aux progressions à thème linéaire ou éclaté (Combettes, 1983). La prise en compte des progressions thématiques, mais aussi des relations de discours, apporterait sans nul doute des données complémentaires, mais complexifierait d'autant la recherche, dont on a vu que même avec une approche limitée elle pose d'assez grandes difficultés

Malgré toutes ses limites, notre étude donne une illustration de la manière dont les CR, en participant de la continuité comme de la discontinuité textuelle, créent 
un maillage local qui n'est ni totalement dépendant, ni totalement indépendant de la structure textuelle. Pour compléter la recherche, on pourrait combiner les différentes approches que nous avons suivies les unes indépendamment des autres. On pourrait aussi prendre en compte l'ordre d'apparition des chaines à l'intérieur des unités structurelles, les positions initiales et finales ayant a priori un statut particulier. Enfin, la cadence des chaînes à l'intérieur des unités mériterait d'être étudiée à son tour. Autant de pistes pour de futurs travaux.

\section{Références bibliographiques}

\section{CFuvre analysée}

Diderot, D. 1984. Essais sur la peinture. Salons de 1759, 1761, 1763. G. May et J. Choulletet (éd.). Paris: Hermann [éd. revue en 2007].

\section{Études}

ADAM, J.-M. 2018. Le paragraphe: entre phrases et texte. Paris: A. Colin.

Ariel, M. 1990. Accessing Noun-Phrase Antecedents. Londres - New York: Routledge.

Benrekassa, G. 1992. Diderot, l'absence d'œuvre. In G. Benrekassa, M. Buffat et P. Chartier (éd.), Études sur "Le neveu de Rameau» et le «Paradoxe sur le comédien» de Denis Diderot: actes du colloque organisé à Paris VII, les 15 et 16 novembre 1991. Cahier Textuel 11. Paris: Université Paris VII: 133-140.

Bessonnat, D. 1988. Le découpage en paragraphes et ses fonctions. Pratiques 57: 81-105.

Charolles, M. 1988. Les plans d'organisation textuelle: périodes, chaines, portées et séquences. Pratiques $57: 3-13$.

Charolles, M. 1995. Cohésion, cohérence et pertinence du discours. Travaux de linguistique 29: 125-151.

CoMBETtes, B. 1983. Pour une grammaire textuelle: la progression thématique. Bruxelles - Paris: De Boeck - Duculot.

Fernandes, A. 2014. Les Salons de Diderot: une chronique de la création artistique. Carnets, Deuxième série (2): 1-11. En ligne à l'adresse suivante: http://journals.openedition. org/carnets/1295.

Givón, T. 1983. An Introduction. In T. Givón (éd), Topic Continuity in Discourse. A Quantitative Cross-language Study. Typological studies in language 3. Amsterdam Philadelphie: J. Benjamins.

HeIDEN, S. 2019. Manuel de TXM. Extension: Annotation URS (Unité-Relation-Schéma), version 1.0. En ligne à l'adresse suivante: https://zenodo.org/record/3267345/files/ txm-manual-urs-extension-v1.0.pdf?download=1.

Heiden, S., Magué, J.-P. et Pincemin, B. 2010. TXM: une plateforme logicielle opensource pour la textométrie - conception et développement. In S. Bolasco, I. CHIARI et L. Giuliano (éd.), JADT 2010 - Statistical Analysis of Textual Data. Proceedings of the 10th International Conference: 9-11 June 2010, Sapienza University of Rome. Milan: Edizioni Universitarie di Lettere Economia Diritto. Vol. 2: 1021-1032. 
Landragin, F., Poibeau, T. et Victorri, B. 2012. ANAleC: A New Tool for the Dynamic Annotation of Textual Data. In N. Calzolari, K. Choukri, T. Declerck, M. Uğur Doğan, B. Maegaard, J. Mariani, A. Moreno, J. Odijk et S. Piperidis (éd.), Proceedings of the 8th International Conference on Language Resources and Evaluation LREC 2012. Paris: European Language Resources Association (ELRA): 357-362. En ligne à l'adresse suivante: http://www.lrec-conf.org/proceedings/lrec2012/pdf/638_Paper.pdf.

Landragin, F. et Schnedecker, C. (éd.) 2014. Langages: Les chaînes de référence 195. Paris: Larousse - A. Colin.

Longacre, R. 1979. The Paragraph as a Grammatical Unit. In J. P. Kimball et T. Givón (éd.), Syntax and Semantics. New York: Academic Press. Vol. 12: Discourse and Syntax: 115-134.

Marandin, J.-M. 1988. À propos de la notion de thème de discours. Éléments d'analyse dans le récit. Langue française 78: 67-87.

Mitterand, H. 1985. Le paragraphe est-il une unité linguistique? In R. Laufer (éd.), La notion de paragraphe. Paris: Éditions du CNRS : 85-95.

Obry, V., Glikman, J., Guillot-Barbance, C. et Pincemin, B. 2017. Les chaînes de référence dans les récits brefs français: étude diachronique (XIII ${ }^{\mathrm{e}}-\mathrm{XVI}{ }^{\mathrm{e}} \mathrm{s}$.). Langue française 195 : 91-110.

Schnedecker, C. 2014. Chaînes de référence et variations selon le genre. Langages 195: 23-42.

Schnedecker, C. et Landragin, F. 2014. Les chaînes de référence: présentation. Langages $195: 3-22$.

VAsAK, A. 2007. La question du genre dans les Salons. In G. Cammagre et C. TAlonHugon (éd.), Diderot, l'expérience de l'art: «Salons de 1759, 1761, 1763» et «Essais sur la peinture». Paris - Poitiers: PUF - CNED : 11-25.

WidlöCher, A. et MATHET, Y. 2009. La plate-forme Glozz: environnement d'annotation et d'exploration de corpus. In Actes de la $16^{e}$ conférence sur le Traitement automatique des langues naturelles - TALN 2009 - Session posters, Senlis, 24-26 juin 2009. En ligne sur HAL : https://hal.archives-ouvertes.fr/hal-01011969. 